Dossier Le naturel et la qualité

\title{
Les Agneaux de Parcours. Rencontres entre producteurs, consommateurs et protecteurs de la nature
}

\author{
Julien Blanc ${ }^{a}$, Marie Rouéb \\ a Docteur en anthropologie de l'environnement, MNHN, Département Hommes, Natures, Sociétés, UMR 5196 \\ Techniques et Culture, Case postale 135, 57 rue Cuvier, 75231 Paris cedex 05, France \\ b Ethnologue, MNHN, Département Hommes, Natures, Sociétés, UMR 5196 Techniques et Culture, Case postale 136, \\ 57 rue Cuvier, 75231 Paris cedex 05, France
}

\begin{abstract}
Entre l'agneau de parcours et l'agneau de bergerie, sur fond de controverse sur le rôle qui doit être réservé au pastoralisme dans la lutte contre la fermeture des milieux, se joue la figure que doit prendre l'élevage à l'heure où tout le monde s'accorde sur la nécessaire qualité du produit livré au consommateur. Les décalages entre les temps du producteur, du boucher et du touriste, les ajustements auxquels les uns et les autres sont contraints, rappellent que la construction de nouvelles normes passe par une confrontation d'intérêts et de savoirs qui engage les acteurs locaux dans une expérience collective de type réflexif.
\end{abstract}

La Rédaction

\section{Mots-clés :}

démarches de qualité ; gestion écologique ; Parc national des Cévennes

\section{Keywords:}

quality labels; ecological management; Parc national des Cévennes
Résumé - Nous nous intéresserons ici au processus de qualification des « Agneaux de Parcours ». Cette filière, née sur le territoire et avec le concours du Parc national des Cévennes, vise à produire un agneau d'herbe tout en assurant une gestion écologique des parcours, souvent négligés par des élevages de bergerie plus productivistes. Les auteurs montrent que le choix d'une filière courte, axée principalement vers les touristes, donne aux bouchers un rôle central de médiateurs. Pourtant, l'inadéquation entre le calendrier des touristes et un calendrier de production qui dépend de la pousse de l'herbe pose un problème majeur. La production d'agneaux plus précoces pour satisfaire la clientèle touristique mettrait en danger l'ensemble du système de production, tandis que les démarches individuelles des éleveurs visant à écouler leur produit quand il est disponible, en particulier par la vente directe, pourraient compromettre la survie $\mathrm{du}$ collectif. Peut-on ajouter, à la tension entre produire et protéger, celle d'une commercialisation locale du produit?

\begin{abstract}
Free-range Lambs. Encounters among producers, consumers and environmentalists. This article considers the quality labelling process of Free-range Lamb. This production-consumption network, originating on the territory and with the support of the Parc national des Cévennes, aims at producing a pasture-raised lamb while ensuring an ecological management of the rangelands that are generally neglected by more production-oriented livestock operations. The authors demonstrate that by opting for a short production schedule that targets tourists as the primary clients, it is the butchers that come to play a central role as mediators. However, the incompatibility of the tourist season with a production schedule that relies upon the greening of pastures, creates a major problem. Advancing lamb production to satisfy the tourist market could undermine the system of production, while the independent initiatives taken by herders to market their product when it comes available, particularly by selling directly to the public, could compromise the survival of the cooperative. It may be that requiring the product to be locally distributed asks too much from a system already strained by the tension between production and protection.
\end{abstract}

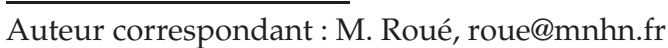


Si les signes de qualité officiels ont une longue histoire - rappelons que les AOC ont été instituées en 1919-, depuis une quinzaine d'années, la qualification des produits alimentaires est devenue à la fois un phénomène de société et l'objet de nouveaux courants de recherche. Le Conseil national de l'alimentation (CNA) lui-même a émis le 26 mars 2002, à la suite d'un processus conduit par son groupe de travail «Notion de qualité», des recommandations à l'attention des filières, des consommateurs et des pouvoirs publics reconnaissant que la qualité, loin d'être un donné, est d'abord une « construction sociale» (Conseil national de l'alimentation, 2002). La politique des signes officiels d'identification de la qualité et de l'origine doit être dynamique et s'adapter aux attentes des sociétés, qui changent au rythme saccadé des crises et de l'émergence de nouveaux registres de préoccupations. Comme le souligne l'économie des conventions (Thévenot, 1995), il n'est plus question aujourd'hui d'une définition de la qualité a priori, puis$\mathrm{qu}^{\prime}$ '« elle est au contraire le produit d'une négociation inachevée qui consiste à tracer un compromis toujours incertain » (Stassart, 2003).

Nous allons ici rendre compte du processus de qualification des "Agneaux de Parcours ». L'originalité de cette petite filière ovine est d'être née d'un partenariat avec le Parc national des Cévennes et de mettre en avant un fort souci environnemental. Nous analyserons la négociation et la mobilisation des réseaux qui ont permis aux bouchers, aux producteurs et aux consommateurs de construire ensemble cette filière. Comme l'ont suggéré les analyses de la sociologie des réseaux et de la sociologie de l'innovation (Callon, 1989; Callon et al., 2000), nous prêterons particulièrement attention aux acteurs et aux objets intermédiaires que sont ici les bouchers et le cahier des charges. Nous devrons, en revanche, laisser de côté la dimension identitaire de l'action collective des éleveurs (Bérard et Marchenay, 1995 ; de Sainte-Marie et al., 1995; Erumel, 2003).

\section{Produire et protéger}

Début 1993, une note interne adressée par la commission Agriculture-Forêt du Parc national des Cévennes (PNC) au directeur suggère qu'il pourrait être intéressant de s'appuyer sur l'image du Parc pour permettre à certains agriculteurs de mieux valoriser le produit de leur travail. L'idée maîtresse est d'utiliser les atouts du Parc, sa renommée internationale, la grande fréquentation de son territoire et de ses alentours ${ }^{1}$, pour promouvoir une agriculture de "productions traditionnelles » alors en réelle difficulté. La commission propose de créer un label Parc

\footnotetext{
1 À l'époque, sur les 600000 visiteurs recensés annuellement en Cévennes, 150000 viennent pour le Parc. De nos jours, celuici annonce une fréquentation annuelle de 800000 visiteurs.
}

national, dont la dénomination serait « Les Authentiques du Parc», et que l'établissement attribuerait à quelques filières et productions agroalimentaires. Pour bénéficier de l'appui du Parc, filières et productions devront contribuer à la protection de l'environnement et à l'entretien des paysages, tout en proposant des produits de très haute qualité. Ce n'est qu'à ces conditions très strictes que le label serait délivré aux agriculteurs. Un certain nombre de filières sont alors pressenties comme pouvant répondre à ces critères : elles vont de la fabrication de jus de pommes de variétés locales à la production de viande de bœuf de race Aubrac, en passant par la culture de châtaignes en Cévennes et l'élevage d'agneaux «haut de gamme", dans des conditions d'excellence écologique. L'opération Authentiques du Parc ne concernera au final que deux produits autour desquels seront développées des filières, les Bœufs de Pâques et les Agneaux de Parcours. La première des deux filières, celle des Bœufs de Pâques, est lancée dès 1995 autour d'une dizaine d'éleveurs du mont Lozère, de quelques artisans bouchers et des restaurateurs de petits bourgs des hautes Cévennes. Son succès encouragera la mise en place, deux ans plus tard, de la filière Agneaux de Parcours, qui répond à la volonté du PNC de promouvoir sur son territoire une agriculture «durable $»^{2}$ face à un phénomène $d$ 'intensificationextensification qui lui pose problème. Un peu partout en zone de moyenne montagne ou en zone sèche, l'utilisation des parcours a été marginalisée par la modernisation de l'agriculture (Chabert et al., 1998). Sur le territoire du Parc comme ailleurs, les éleveurs favorisent l'utilisation de ressources non saisonnées, réserves alimentaires constituées à partir des surfaces cultivées, au détriment des ressources à disponibilité saisonnière, les parcours (Lardon et al., 1995). Rappelons que les parcours sont des surfaces dédiées au pâturage des animaux et regroupent des formations herbacées et buissonnantes que l'on distingue des prairies naturelles, milieux à plus forte productivité qui peuvent être fauchés. Le Parc considère que ces évolutions sont préjudiciables à l'environnement et au maintien de la biodiversité (PNC, 2000). Sur les terres cultivées, les pratiques visant à l'accroissement des rendements augmentent l'utilisation d'engrais et de produits phytosanitaires qui polluent les nappes phréatiques. Les techniques de conservation des fourrages adoptées par les éleveurs à la fin des années 1980 (ensilage et enrubannage), si elles leur permettent de faucher plus tôt leurs prairies, empêchent par contre la nidification de certaines espèces d'oiseaux protégés, tel le busard Saint-Martin. La propension des éleveurs à accroître leurs terres cultivées provoque également la disparition,

\footnotetext{
${ }^{2}$ Dans son rapport d'activité 2003, l'opération Agneaux de Parcours s'intègre notamment à un volet développé par le Parc afin de «[v]aloriser l'origine Parc national des produits du territoire issus d'un mode de production dit durable » (Rapport d'activité 2003, Orientations stratégiques, 11).
} 
ou du moins la réduction, de milieux à valeur dite patrimoniale $^{3}, c^{\prime}$ est-à-dire de milieux où se développent des espèces animales et végétales protégées que le Parc a pour vocation de conserver.

Le PNC a inscrit la protection des milieux ouverts comme une de ses priorités et entend, à cet effet, favoriser les éleveurs qui pratiquent une " gestion écologique », en particulier le collectif Agneaux de Parcours. Pourtant, de plus en plus de chercheurs mettent en doute la capacité des éleveurs à garder les milieux et les parcours « ouverts" en y faisant pâturer leurs troupeaux (Rousset et Lepart, 1999). Ils nous rappellent que les éleveurs, sans le recours à des opérations mécaniques de débroussaillement, ne peuvent renverser un processus aussi complexe, dont les causes tiennent à la fois à des phénomènes naturels, aux modifications des pratiques agricoles et à l'évolution démographique de la société locale (Friedberg et al., 2000 ; Lepart et al., 2000). La perception de l'ouverture ou de la fermeture du paysage n'est pas basée sur des faits, mais sur des représentations qu'il convient d'analyser en tant que telles. Le renversement du discours qui, après avoir, au XIXe siècle, fustigé les éleveurs pour leurs pratiques qui auraient été à l'origine de la déforestation, les condamne, au début du XXI ${ }^{\text {e }}$ siècle, pour leur incapacité à maintenir le paysage ouvert, est en effet d'une grande ironie.

Sans aller plus avant dans ce débat, notons que les éleveurs de l'association Agneaux de Parcours mobilisent plus largement les parcours que les éleveurs d'agneaux de bergerie. Ils ont donc au moins une action relative, sinon absolue, sur leur entretien. Pour le Parc, cette opération, qui vise à replacer « les parcours au cœur du système de production ", a donc valeur d'exemple, même si elle ne peut résoudre à elle seule toutes les contradictions de l'agriculture contemporaine.

Il était logique que la filière Agneaux de Parcours s'oriente, pour sa commercialisation, vers un circuit court, qui diminue les coûts et la consommation d'énergie, en particulier pour le transport, et s'inscrit dans une démarche de respect de l'environnement ${ }^{4}$. La commercialisation de ce produit local sur le territoire même du Parc procède d'une logique de valorisation d'un mode

\footnotetext{
${ }^{3}$ Soit qu'il s'agisse de milieux abritant des populations d'espèces végétales protégées, soit même qu'il s'agisse de milieux inscrits à la directive Habitats.

${ }^{4}$ Un article de C. Sunt intitulé « Réinventer les circuits courts : une nécessité pour la bio ", paru dans les revues Nature et Progrès ( $n^{\circ} 40$, mars-avril 2003) et Fruits Oubliés ( $\left.n^{\circ} 30,4 / 2002\right)$, dont l'auteur est président, puis sur le Web, critique la «bio industrielle » prisonnière du marché. Il propose de développer des marchés de proximité pour « relocaliser » l'économie et cite Terroir Direct, qui propose à ses adhérents d'acheter chaque semaine un colis de produits fermiers et artisanaux « dont $50 \%$ issus de l'agriculture biologique». En 2003, 55 agneaux de parcours ont été commercialisés par cette association, et 130 en 2004.
}

de production et d'un pastoralisme seul à même de perpétuer des paysages qu'il a contribué à créer.

La philosophie de la filière implique que l'élevage, la vente et la consommation se déroulent sur le territoire du Parc. Impliquant des élevages autonomes et économes, qui dépendent pour l'essentiel de ressources naturelles et humaines disponibles localement, la majeure partie de la valeur générée par la filière est réinvestie au niveau local. La filière s'inscrit ainsi dans une démarche de « développement local intégré » (Long et Van der Ploeg, 1994) et peut être considérée comme exemplaire pour la mise en œuvre d'un développement durable.

Pourtant, nous verrons que le choix d'une filière courte peut conduire à quelques paradoxes quand les clients locaux sont en majorité des touristes, dont le cycle de migrations n'est pas en phase avec la production des agneaux qu'ils désirent consommer. Ce problème est particulièrement complexe puisque le processus de production, refusant toute artificialisation, $\mathrm{s}^{\prime}$ inscrit dans un cycle qui dépend de conditions naturelles, la pousse de l'herbe, sur lequel l'éleveur ne peut influer.

\section{Naissance d'une filière au Parc national des Cévennes}

Née tout d'abord d'une rencontre entre deux individus, un éleveur et un employé du Parc national des Cévennes, la filière Agneaux de Parcours a été, par la suite, pensée et mise en place, à partir d'un noyau d'éleveurs qui ont rapidement vu l'intérêt de la coopération proposée par le Parc pour la valorisation de leur produit. Les études de faisabilité commandées par le Parc à $1^{\prime}$ ISARA ${ }^{5}$ étaient encourageantes, puisqu'elles confirmaient de réelles potentialités en termes de commercialisation. Un agneau de saison élevé à l'herbe des pâturages et au lait maternel, régulièrement conduit à l'air libre, voire élevé en plein air intégral, et subissant peu d'interventions prophylactiques, permettait aux éleveurs de revendiquer « une production au naturel ». L'appellation «Parc national des Cévennes », en gagnant la confiance des consommateurs, amène une meilleure commercialisation sans transformer le mode de production déjà pratiqué par les éleveurs.

La première démarche pour les éleveurs et le Parc fut de s'atteler, en 1996, à la rédaction d'un cahier des charges qui conviendrait au mieux à chacun. Cet engagement fut, de fait, peu contraignant pour les éleveurs, car il cautionna des pratiques d'élevage qui correspondaient déjà largement aux leurs et satisfaisaient la demande du Parc en termes de gestion écologique. Aucune standardisation ne fut imposée. Notons, par exemple, que les éleveurs qui s'associaient n'élevaient pas les mêmes races. La Blanche

\footnotetext{
${ }^{5}$ Institut supérieur d'agriculture et d'agroalimentaire RhôneAlpes.
} 
$\mathrm{du}$ Massif central dominait, mais des agneaux issus de brebis Black Face et Clun Forest, ainsi que de Rouge du Roussillon ou de race Lacaune étaient également présents dans leurs troupeaux. Le groupe des éleveurs était d'une grande diversité, comprenant des néoruraux et des éleveurs originaires de la région, installés en des lieux éloignés au sein du Parc national des Cévennes : causse Méjan, mont Lozère et montagnes cévenoles. Ce qui les réunissait, c'était une volonté d'autonomie par rapport à des filières plus productivistes, une détermination de produire au moindre coût et, pour le noyau dur, de fortes convictions environnementales qui les amenaient à produire des agneaux d'herbe.

Une fois le cahier des charges de l'association Agneaux de Parcours rédigé, il fallut intégrer à la démarche des distributeurs pour construire un réseau de distribution avec les bouchers et les restaurateurs locaux désireux de participer à l'opération. À la suite de nouvelles négociations entre les éleveurs et ces derniers, portant essentiellement sur les prix, les modalités de livraison et l'organisation du fonctionnement de la filière à l'aval de la production, une association de loi 1901 fut créée en juillet 1997. Elle regroupe les éleveurs, qui en constituent la majorité des membres, les distributeurs et le Parc, dont la fonction est essentiellement de veiller au respect des clauses du contrat, sans pour autant intervenir directement sur la dynamique de l'opération. Cela fait aujourd'hui sept ans que ce petit groupe d'éleveurs a maintenu la même structure associative, soutenue par le Parc qui, volontairement, reste en retrait, dans un rôle de facilitateur. Le label initialement prévu n'a donc jamais été déposé, et les Agneaux de Parcours sont toujours, d'une part une association loi de 1901, d'autre part un projet du Parc 6 .

Dans le même temps, on a vu les parcs régionaux faire le choix de déposer une marque collective PNR protégée et propriété de l'État, qui en confère la gestion à chaque parc. Dans ce cadre, l'attribution de la marque reste une prérogative de chacun des parcs. En fonction de la nature du marquage, organisé en trois secteurs : produits agricoles ou artisanaux, prestations touristiques et savoirfaire, il est alors fait mention de «Produit du », « Accueil $\mathrm{du}$ » ou «Savoir-faire $d u$ », suivie du nom du parc. Des viandes bovines et ovines sont, par exemple, soutenues dans les Parcs du Vercors, de la Chartreuse, du Morvan, et dans le Haut-Languedoc. D'autres opérations, comme celle du PNC, valorisent un développement durable qui protège la biodiversité : le jus de pomme du PNR du Vexin français vise à valoriser un produit et à protéger un milieu favorable à la chouette chevêche. Ainsi, l'initiative

\footnotetext{
${ }^{6}$ Le programme d'aménagement du Parc pour 2000-2006 exprime ainsi son soutien aux Agneaux de Parcours dans son orientation stratégique 11 : «Valoriser l'origine Parc national des produits du terroir issus d'un mode de production dit durable».
}

du PNC s'inscrit dans, et peut-être même anticipe, une tendance générale au sein des parcs français, qu'ils soient nationaux ou naturels régionaux.

Il peut paraître étonnant que le PNC et les éleveurs ne se soient pas engagés dans une démarche de reconnaissance officielle autre que celle du Parc. De fait, la question a été plusieurs fois abordée par les membres du réseau Agneaux de Parcours. La demande d'AOC a été abandonnée en raison du volume de production qu'elle impliquait. L'indication géographique protégée (IGP) a été envisagée par les éleveurs en relation avec la chambre d'agriculture. Ils y ont renoncé, craignant une démarche à la fois trop coûteuse et trop normée, qui obligerait les éleveurs à travailler avec une seule race ovine, la Blanche du Massif central. Une certification de conformité de produit (CCP) a également été envisagée, puis abandonnée pour des raisons similaires de coût et de contraintes. Pendant une année, en 2003, l'association a demandé à une filiale de la chambre d'agriculture de contrôler le respect du cahier des charges des adhérents. Cette démarche avait été motivée par l'inquiétude des consommateurs face au retour de la fièvre aphteuse, mais également par une demande récurrente du PNC. Pourtant, les éleveurs ont trouvé ce contrôle trop coûteux et ne l'ont pas renouvelé. Le président de cette petite association souligne l'impossibilité, pour une petite structure, de monter des dossiers et constituer des réseaux qui demanderaient à être pris en charge par des professionnels de la mise en marché.

Plusieurs des éleveurs ont, à titre individuel, engagé une reconversion à l'agriculture biologique. Après une année ou deux de fonctionnement, leur discours reflète une désillusion. L'avantage économique principal de cette reconversion semble être avant tout les crédits touchés pour la mener à bien. Par la suite, les surcoûts impliqués par une production bio se révèlent souvent trop élevés. Un éleveur affirme, par exemple, que vendre sa production sous un label biologique - ce qui nécessite un matériel spécifique de culture et l'achat de produits chers -, non seulement ne lui rapporte rien, mais lui coûte 4000 euros par an. Il pense donc abandonner, tout comme un autre qui, après sa reconversion, envisage de faire comme si de rien n'était et de continuer à développer une relation de proximité avec une clientèle privée, à qui il vend $\mathrm{du}$ « bio de confiance non labellisé » et qui n'a pas besoin de l'étiquette pour avoir confiance dans sa production.

Actuellement, l'association, qui compte une dizaine d'éleveurs environ, s'engage vers le simple dépôt d'une marque auprès de l'Institut national de la propriété industrielle (INPI) afin, tout au moins, de protéger son produit. Elle vient de soumettre auprès de la Communauté européenne, via le programme Leader, une demande de subvention pour financer un contrôle régulier de son cahier des charges. Le Parc accompagne également un 
programme qui vise, sur cinq ans, à améliorer la traçabilité et la qualité du produit.

\section{Des agneaux qui poussent en même temps que l'herbe}

Qu'est-ce qu'un agneau de parcours? C'est tout d'abord un agneau nourri du lait de sa mère et de l'herbe des pâturages. Sur le territoire du Parc, de tels élevages sont nettement minoritaires, la plupart des éleveurs ayant opté pour l'élevage d'agneaux en bergerie. Selon le responsable du Service de protection et d'aménagement du territoire pour le Parc, moins du tiers de l'ensemble des élevages ovins en activité dans le Parc national des Cévennes produit ses agneaux à l'herbe. La plupart de ces élevages à l'herbe sont situés dans les montagnes cévenoles et sont d'une taille plus modeste que leurs analogues de bergerie. Sur le causse Méjan, par exemple, où l'élevage est particulièrement dynamique et où les structures sont de taille importante, l'agneau $\mathrm{d}^{\prime}$ herbe est minoritaire (7 élevages sur $30^{7}$ ).

Si les agneaux de parcours peuvent passer la nuit dans une bergerie, ils sont le plus souvent à l'air libre, sur les pâturages et notamment sur les parcours dont ils se nourrissent. Bien qu'essentiellement nourris à l'herbe, ils peuvent recevoir une «complémentation » alimentaire soumise à une double restriction :

- quantitative tout d'abord, les apports alimentaires ne devant pas, selon le cahier des charges, «dépasser 20 kilogrammes de céréales ou d'équivalents » par agneau, alors que la complémentation des agneaux élevés à l'herbe varie en France entre 20 et $120 \mathrm{~kg}$ par animal, tous produits confondus (Prache et Thiérez, 1990);

- qualitative ensuite, puisque seuls sont admis des compléments alimentaires locaux à base de châtaignes, glands ou céréales produits sur la ferme ou sur une ferme voisine du PNC, le recours à des aliments à base de céréales issues de l'agriculture biologique, sans indication de provenance, étant toléré. Cette restriction vise à appuyer l'ancrage territorial de la production.

Un agneau de parcours est un produit de saison. Le cahier des charges stipule que les agneaux doivent naître entre le $1^{\text {er }}$ février et le 31 avril. Lorsque les agnelages sont pratiqués dans cette fourchette calendaire, selon la formule des éleveurs, ils " poussent en même temps que l'herbe ». Cette spécification vise un agnelage unique et une concordance entre le cycle de reproduction des animaux et celui de la production herbacée. Si la date d'ouverture de la période d'agnelage est fixée si tôt, c'est pour que des élevages situés en des lieux où le démarrage de

\footnotetext{
${ }^{7}$ Dont six sont intégrés à la filière Agneaux de Parcours, le septième vendant ses agneaux très jeunes, et non engraissés.
}

la végétation est précoce - par exemple, les fonds des vallées cévenoles - puissent s'intégrer à la filière.

Enfin, les éleveurs s'engagent à respecter des pratiques naturelles concernant la santé de leurs agneaux. Le cahier des charges, non seulement interdit le recours à « une alimentation médicamenteuse » et aux traitements de synchronisation des chaleurs («la pose d'éponge»), mais limite également les interventions thérapeutiques sur les agneaux et leurs mères.

\section{À la recherche d'agneaux plus précoces}

« La vente des agneaux de parcours est autorisée du $1^{\text {er }}$ juin au 15 novembre ${ }^{8} »$. C'est ce que stipule le cahier des charges Agneaux de Parcours. Cependant, le premier lot d'agneaux n'est généralement disponible que fin juin, et ce n'est qu'à la mi-juillet que la production suffit à approvisionner les bouchers de la filière. Dans les premiers temps de l'opération, les bouchers faisaient pression sur les éleveurs, notamment sur les caussenards, pour obtenir des agneaux plus rapidement. En effet, si la grande majorité des estivants n'arrive qu'au début du mois de juillet, dès le $1^{\text {er }}$ juin, quelques visiteurs constituent l'avant-garde des consommateurs potentiels. La date d'entrée de la période de vente de la charte avait été « pensée » en fonction de la demande plutôt que de la capacité de production.

À la mi-juillet, les agneaux sont pourtant encore un peu «justes », c'est-à-dire trop légers. Les éleveurs, face à la forte demande estivale des bouchers liée à l'afflux des clients, sont alors conduits à leur proposer des agneaux à l'engraissement insuffisant. Ce problème de déséquilibre entre l'offre et la demande perdure chez certains éleveurs du causse Méjan jusqu'à la fin du mois d'août. «On ne peut pas en fournir plus de 40-50 - explique l'un d'entre eux - parce que, dans le mois d'août, on n'a pas plus d'agneaux qui sont prêts. C'est les meilleurs, là, qui s'en vont, et encore ils sont justes. C'est des agneaux qui gagneraient à rester un mois de plus. »

Chez les éleveurs situés en Cévennes ou sur le flanc du mont Lozère, à moindre altitude, les agneaux sont prêts plus tôt, début juillet ou un peu plus tard ${ }^{9}$. Un seul éleveur réalise un agnelage précoce et peut fournir des

\footnotetext{
8 Cette période est celle mentionnée par les cahiers des charges rédigés entre 1997 et 2002. Depuis, la période de vente autorisée a été décalée et ne débute qu'au $1^{\mathrm{er}}$ juillet pour finir au 31 décembre.

${ }^{9}$ En attendant, les brebis sont soit maintenues en bergerie, affouragées et complémentées en céréales, soit menées sur des prairies dont la pousse est plus précoce en raison tout à la fois de la fumure qui y est apportée, de leur situation en fond de cuvette (mieux protégées) et de leur composition en légumineuse dominante, famille dont la pousse serait plus précoce. Les agneaux, quant à eux, ne consomment alors encore que du lait maternel.
} 
agneaux plus tôt dans la saison, car sa ferme est située à une altitude inférieure à celle des autres élevages de la filière implantés sur le causse Méjean. L'hiver moins rigoureux et le printemps plus précoce favorisent un démarrage de la végétation et de l'herbe beaucoup plus tôt dans la saison. Dans les Cévennes, les châtaigneraies assurent en outre des ressources hivernales non négligeables qui permettent d'avancer l'agnelage. En règle générale, pourtant, les éleveurs de la filière n'ont d'autre choix que de coordonner leurs agnelages avec la pousse de l'herbe. Ils programment les naissances trois semaines à un mois avant que l'herbe ne soit disponible sur les pâturages.

Aujourd'hui, les bouchers sont mieux au fait de ces contraintes naturelles. Dès lors que leur objectif est de commercialiser un agneau «naturel», ils acceptent ces inconvénients inhérents au produit et indépendants de la volonté des éleveurs. L'un d'entre eux s'exprime ainsi : «On aimerait qu'ils puissent nous ravitailler un peu plus tôt dans l'année, vers le 15 juin plutôt que le 15 juillet, mais bon, suivant les secteurs, si c'est le causse ou les Cévennes [... ] ils font ce qu'ils peuvent, quoi... »

Leur grand espoir, compte tenu des contraintes naturelles, est d'intégrer plus d'éleveurs cévenols à la filière, même si, les exploitations dans les Cévennes étant de petite taille, la proportion d'agneaux mis en vente par ces nouveaux adhérents risquerait de n'être pas très significative : "Il y a des gars des Cévennes qui m'ont contacté, alors peut-être j'arriverai à en avoir plus tôt ; parce que, en Cévennes, ils arrivent à faire mettre bas plus tôt. . . le climat n'est pas le même, ils vont déjà dehors alors qu'au même moment sur le causse. . . »

Pour les éleveurs, deux stratégies sont possibles pour obtenir des agneaux prêts à la vente plus tôt, plus gros et mieux finis aux mois de juillet et août : avancer la date de l'agnelage ou accélérer la croissance des agneaux. Elles ont toutes deux été tentées par les éleveurs avec un succès inégal.

Pour accroître la vitesse de croissance de ces agneaux afin qu'ils soient prêts à la vente en trois mois, la marge est étroite : il faudrait mieux les nourrir, ce qui se révèle difficile en respectant le cahier des charges. La contrainte pèse à la fois sur la nécessité d'utiliser les parcours, et non les prairies qui permettent un engraissement rapide, et sur la limite du recours à la complémentation.

Un des éleveurs obtient des vitesses de croissance et d'engraissement plus rapides en se débarrassant d'un de ses agneaux «nés doubles », afin d'assurer un meilleur allaitement et une meilleure croissance à son jumeau. Parce qu'il vend certains de ses agneaux « légers » vers un mois, par le biais d'une autre filière, il parvient à mieux valoriser ceux qu'il garde : une partie de ses agneaux sont prêts dès trois mois et la totalité à l'âge de six mois. Mais il lui faut également complémenter plus que les autres éleveurs, et frôler la limite supérieure de complémentation autorisée par la filière.

Depuis un à deux ans, grâce aux nouveaux débouchés que leur propose l'association Terroir Direct, certains éleveurs ont commencé à valoriser l'un des jumeaux en le vendant très jeune comme agneau de lait. Cette démarche représente pour eux un progrès certain par comparaison à celle que nous venons de décrire, qui implique de vendre à bas prix un des jumeaux au groupement de producteurs local.

Un autre éleveur a tenté l'expérience d'avancer les agnelages de près d'un mois, mais il a constaté que cette modification portait préjudice à l'ensemble de ses résultats, car, selon lui, le foin n'a pas la «force» de l'herbe verte. Les brebis ont par conséquent moins de lait et les agneaux, qui ne disposent pas d'herbe dès qu'ils sont aptes à en manger, sont plus faibles et sujets au parasitisme. Par ailleurs, le savoir des éleveurs pointe un autre inconvénient à l'agnelage précoce. Lorsque l'on met les agneaux le plus tôt possible à l'herbe, dès qu'elle commence à croître, cette consommation très précoce sur les parcours «casse » l'herbe et est préjudiciable à sa pousse ultérieure.

Ce système d'élevage, dont une des caractéristiques essentielles est une saisonnalité marquée - l'agneau devant pousser en même temps que l'herbe -, répond donc difficilement aux contraintes de sa mise en marché. La filière courte choisie visant un public d'estivants, qui arrivent et repartent trop tôt pour l'agneau, le dispositif fonctionne cependant, mais avec quelques désagréments pour tous. Pour sortir de ces contradictions, plusieurs éleveurs développent leur propre clientèle, livrant sur Montpellier ou même sur Paris. C'est alors la clientèle, et non l'agneau, qui est disponible en grand nombre et toute l'année. Mais on n'est plus dans la représentation de l'idéal militant : produire et vendre localement.

\section{L'agneau de parcours, produit d'appel pour les bouchers}

Chez les bouchers, toute dimension idéologique ayant trait à la dimension environnementale de l'opération semble absente de leur discours. Seul l'un d'entre eux a laissé entendre son adhésion de vues à une telle entreprise, ou tout du moins à l'engagement idéologique de certains des éleveurs de l'opération. Son évocation, critique, des élevages où "l'agnelage se fait en hiver dans des hangars » confirme que les éleveurs avec qui il travaille lui plaisent parce qu'ils travaillent plus «naturellement » et ne sont pas des "productivistes ». Cependant, à l'instar des autres bouchers, le discours environnementaliste du Parc ne semble pas l'avoir beaucoup marqué : «À la première réunion, le Parc nous avait expliqué la biosphère et tout ça, là, l'environnement, le nettoyage », 
nous dira-t-il. De fait, les bouchers ne semblent pas avoir été particulièrement accrochés par la vocation de gestionnaires environnementaux que le Parc cherche à associer aux élevages de la filière.

Ce n'est pas non plus parce qu'ils vendent cet agneau plus cher qu'un autre, de facture plus anonyme, que les bouchers ont décidé de participer à cette opération. Leur motivation est, en fait, liée à l'avantage global qu'ils tirent à vendre ce produit en termes d'image. Tous déclarent vendre plus d'agneaux qu'auparavant. En bref, vendre des agneaux de parcours restaure la confiance, attire la clientèle et accroît la fréquentation de leur boucherie. Trois des quatre bouchers de l'opération vendent également du bœuf de Pâques. Proposant ainsi de l'agneau et du bœuf de "qualité », ils gagnent en réputation auprès de leur clientèle : «Il fait de la bonne viande, je ne vais qu'ici », nous confiera un client estival. Ce gain d'image auprès de leur clientèle, plusieurs bouchers l'avaient subodoré : "On savait que ça allait marcher », nous dira l'un d'eux. Car, plus globalement, adhérer à une telle démarche joue sur un ressort qu'ils connaissent bien : «Dans notre métier, maintenant, il faut se démarquer, il faut trouver un petit truc pour se démarquer. »

Pour faire face à la concurrence, qu'elle vienne des boucheries environnantes fréquentées par le même type de clientèle, ou des supermarchés qui attirent une quantité croissante de consommateurs, il faut se démarquer, communiquer sur l'origine identifiée et saine de son produit, par contraste avec le tout-venant de la production industrielle dangereusement anonyme : "C'était juste après la vache folle; on avait pris une grosse gifle, et il fallait redonner un peu de confiance à nos clients; ils ont eu très peur avec cette histoire de vache folle. »

L'avantage que tirent les bouchers de la vente de ce produit, et qui explique en grande partie leur adhésion à l'opération, repose donc sur son identité, qui contribue à établir une relation de confiance avec les consommateurs. Les agneaux de parcours constituent pour eux un produit d'appel dont le crédit rejaillit sur l'ensemble des transactions.

Nous l'avons vu tout au long de cet article, c'est sur les bases d'un lien fort avec le Parc national des Cévennes que fut engagée l'opération Agneaux de Parcours. Des éleveurs qui, du point de vue du Parc, pratiquent «bien", fournissent à des bouchers des agneaux que ces derniers vendent sous le sceau du Parc national des Cévennes tout au long de la saison de production. Les visiteurs du Parc étant a priori particulièrement sensibilisés par un produit issu d'élevages situés au cœur de cet espace protégé et de pratiques au plus «naturel » qu'il soit, c'est en fonction de cette cible que furent réalisées les brochures promotionnelles et choisie une telle dénomination pour ce produit. Comme nous le préciseront les bouchers, l'image véhiculée par le Parc auprès de ses visiteurs est à la source de leur désir de consommer un agneau «Parc»: «Tout de suite on a dit oui, parce que c'étaient des bons produits et puis surtout pour pouvoir se servir aux alentours de Florac. Les gens, c'est ce qui est important pour eux, que la marchandise vienne le plus près possible $\mathrm{d}^{\prime}$ ici. Et surtout les touristes, ici ils viennent pour manger $\mathrm{du}$ bon, ils ne viennent pas pour manger ce qu'ils trouvent toute l'année. » «Mais c'est vrai que rien que de savoir $\mathrm{d}^{\prime}$ où il vient, ils [les consommateurs] le trouvent meilleur, quoi... c'est un peu psychologique... [Rires.]».

Ce lien est assuré par la dénomination Parc, mais aussi par des brochures promotionnelles qui font correspondre l'un et l'autre par le biais, notamment, des notions de «naturel » et de «sain », communes à ces deux " produits». En 1997, l'enquête réalisée auprès des futurs consommateurs d'agneaux de parcours ${ }^{10}$ et visiteurs du Parc avait d'ailleurs montré que ces derniers se faisaient une image du Parc national des Cévennes tout à fait en accord avec celle que cherche à développer l'établissement public, même si elle est assez loin de la réalité du lieu : «La majorité des touristes interrogés associe l'image de la Lozère à celle d'une région protégée où l'agriculture reste traditionnelle, s'effectuant dans le respect de la nature et de l'animal. Ils n'ont pas conscience que dans cette région aussi, l'intensification est de plus en plus présente » (Aupetitgendre et Beisson, 1997, p. 35).

Pourtant, cette viande présente en outre une qualité organoleptique certaine, et supérieure à celle de la plupart des autres agneaux vendus. Ce goût "meilleur», que les bouchers associent aux pratiques d'élevage spécifiques aux agneaux de parcours et à la façon dont ces animaux sont nourris, participe à l'engouement des consommateurs : "Au niveau goût, ça n'a rien à voir ; nous, on a des clients qui ne veulent que de l'agneau de parcours. On n'a que des compliments sur la viande. »

Les bouchers affirment que c'est, en premier lieu, $l^{\prime}$ allaitement des agneaux par leurs mères ${ }^{11}$ et, dans une moindre mesure, l'alimentation à base d'herbe qui donnent son goût à cette viande. Par opposition aux agneaux de bergerie, les faibles proportions d'aliments concentrés et de céréales et, corrélativement, les fortes proportions d'herbe dans l'alimentation des agneaux de parcours ont également une influence certaine sur le goût, même si elle reste minime par rapport à celle de l'allaitement.

\footnotetext{
10 À la demande du Parc et des éleveurs, avant de lancer la démarche.

11 Par contre, les éleveurs de bergerie pratiquent en général un sevrage précoce de leurs agneaux à un mois et demi, sauf ceux d'Elovel, avec laquelle les bouchers locaux ne travaillent pas. L'allaitement constitue, pour eux, une pratique propre à l'élevage des agneaux de parcours.
} 


\section{Les bouchers au cœur de la négociation de la qualité du produit}

L'autre atout important de cet agneau, ce sont les bouchers, qui peuvent convaincre le consommateur de sa qualité exceptionnelle. Directement au contact des consommateurs, ils contribuent à renforcer le lien entre le lieu de production, les conditions d'élevage et les caractéristiques de la viande. Les propos qu'ils tiennent à leurs clients emportent leur adhésion. Ils sont à même de vanter ces agneaux élevés à l'air libre, avec leurs mères et sur de grands espaces préservés, et de convaincre les clients du caractère sain du produit.

En assistant à ces explications, nous avons pu relever les arguments utilisés par un boucher face au consommateur. Artisans bouchers, de campagne, ils n'hésitent pas à affirmer qu'ils effectuent leurs achats directement auprès des éleveurs, ce qui leur confère un statut d'expert aux yeux de leur clientèle. C'est là un ressort essentiel de la confiance du client envers le boucher et, par-delà, de son attrait pour le produit : « Les gens, ils nous font confiance ici, parce qu'on travaille beaucoup avec le monde paysan, on va abattre nos bêtes et tout ça, donc on n'a pas les mêmes problèmes qu'à Paris, que sur les grandes villes... On voit pratiquement toutes nos bêtes vivantes. »

Pourtant, les bouchers nous le diront clairement au cours de l'enquête, le produit n'est pas, pour eux, sans défauts, loin de là. On pourrait même affirmer, en résumant leurs propos, qu'il n'est jamais comme il faut, quelle que soit la période de vente considérée.

Tout d'abord, contrairement aux agneaux élevés en bergerie, qui constituent la majorité des agneaux produits localement, les modalités de production de l'agneau de parcours ne permettent pas d'en faire un produit standardisé. Les pratiques retenues dans le cahier des charges en font un produit dont les caractéristiques ne peuvent être disjointes des conditions «naturelles » de la production. En bergerie, au contraire, quelle que soit leur saison de production, tous les agneaux peuvent, si l'éleveur le désire, présenter les mêmes caractéristiques (en âge, poids, couleur de viande, gras, goût... ). L'alimentation des agneaux de bergerie, qui pour la plupart ne voient pas le jour et ne se déplacent pas, est constituée parfois de lait maternel et dans tous les cas de céréales, de foin et d'aliments achetés dans le commerce. Elle est largement maîtrisée par l'éleveur. Certes, l'alchimie alimentaire est complexe, et les agneaux seront plus ou moins gras lors de leur vente. Mais, si l'éleveur le désire, ils feront tous à peu près le même poids et seront tous vendus entre 3 et 4 mois. La couleur de leur viande sera la même et son goût peu variable, bien que les variations de niveau de gras aient une influence certaine. Toutes ces caractéristiques normatives en font des produits dont la vente est aisée pour les bouchers.
Par opposition, les agneaux de parcours sont très peu complémentés. Leurs modalités d'élevage, qui s'appuient sur le rythme des saisons, et donc sur les variations saisonnières de la production, entraînent une variation importante du produit. Le poids à la vente, par exemple, varie entre 14 et $21 \mathrm{~kg}$, tandis que l'âge des animaux tués s'étale de 3 à 8 mois. Les bouchers considèrent qu'alors que les agneaux très jeunes sont souvent trop petits début juillet - quand commence la saison touristique -, en fin de saison, ils deviennent trop gros. Selon la saison, des clients rechignent face à des côtelettes sur lesquelles il n'y a pas grand-chose à manger ou, à l'opposé, sur des gigots par trop imposants et, par conséquent, trop coûteux. De plus, du fait de son mode de production, la couleur, le goût et le niveau de gras de la viande évoluent au fil du temps. D'une couleur claire et d'un goût très fin assurés par la jeunesse des agneaux et grâce au lait maternel qui constitue encore une grande partie de leur alimentation en début de saison, la viande passe à une forte coloration à partir de la mi-août et son goût devient nettement plus prononcé. À cette époque, en effet, les agneaux déjà âgés ne tètent quasiment plus, voire plus du tout, car les éleveurs les ont sevrés. Les bouchers savent que l'âge de l'animal, la quantité de lait ingérée et la consistance de $l^{\prime}$ herbe qu'il consomme influencent directement la couleur et le goût de la viande. De fait, la production des agneaux de parcours étant strictement saisonnalisée, ces trois facteurs, bien qu'étant considérés chacun par les bouchers et les agronomes comme ayant une influence propre sur la couleur de la viande, sont étroitement dépendants. Les agneaux naissant au printemps et étant commercialisés à partir de la mi-juillet jusqu'à la fin novembre, c'est la saisonnalité de la production qui pilote les autres facteurs, le sevrage des agneaux tel que le pratiquent les éleveurs étant naturel ou presque, à 5-6 mois.

Le goût, la couleur et le poids des carcasses varient donc au cours de la saison de production. Mais, alors que goût et couleur tendent, au fil du temps, à s'éloigner de plus en plus des attentes des consommateurs, le poids de la carcasse, lui, s'améliore. Les bouchers doivent donc commercialiser un produit qui, à leurs yeux, n'est jamais parfait : "On a tous le même problème. Au départ ils sont trop petits et la couleur est bonne, et après, quand on avance dans la saison, la couleur devient un peu plus rouge et au niveau poids ils sont bons... » Ils ont un rôle essentiel dans le processus de construction de la qualité du produit, celui de faire accepter aux consommateurs sa variabilité. Proches du lieu de production de l'agneau qu'ils distribuent, ils se présentent aux yeux des touristes et des habitants permanents des Cévennes comme des experts, à même d'attester de la qualité de leur produit. Pourtant, leur propre perception de celleci n'est pas exempte de représentations. Reprenons, par exemple, le cas de la couleur. $\mathrm{Si}$, pour les bouchers comme pour leurs clients, la couleur blanche est gage de qualité, 
c'est seulement parce qu'elle est la norme : la plus grande partie des agneaux étant élevés en bergerie, au lait maternel mais aussi à l'aide de compléments alimentaires, leur viande n'acquiert pas la coloration rosée que prend celle des agneaux d'herbe. Il est donc paradoxal que la couleur blanche de la viande soit devenue aux yeux de tous un gage de qualité.

Face à la réticence des clients, les bouchers sont régulièrement conduits à argumenter sur les raisons de cette coloration plus prononcée et non habituelle de la viande. Voici les arguments utilisés par l'un d'eux : "Ce n'est vraiment pas du tout la même marchandise; parce que d'abord, d'habitude, les agneaux ne voient pas trop le jour, ils ne se déplacent pas, ils restent toujours sur place, ils grossissent plus vite. Ces agneaux-là, il faut 4, 5, 6 mois. Du coup la viande est différente, c'est de l'agneau qui est à l'air libre, qui tète, qui mange de l'herbe, c'est le broutard... » Un autre boucher, en évoquant ce problème, nous dira à propos de sa clientèle habituelle qu'il « faut leur expliquer... mais maintenant ça va, ils savent ». Les bouchers sont à même de convaincre leur clientèle que cette couleur n'est pas délétère pour la qualité de la viande, voire qu'elle peut être considérée comme un critère de qualité. Ces convictions sont-elles, pour eux, aussi saisonnières que la production qu'elles valorisent, ou entraînent-elles un changement radical de leurs représentations de ce qu'est une bonne viande d'agneau?

\section{La construction sociale d'un produit de qualité}

Ce que nous avons tenté de montrer ici, en décrivant une filière qui articule production et protection de la nature, c'est que la négociation entre acteurs conduit parfois à des injonctions contradictoires. Négociation entre des éleveurs qui ont des objectifs de production et un Parc qui leur demande d'être aussi des protecteurs des espèces menacées. Négociation entre des bouchers qui apprécient de vendre un produit de qualité, mais aimeraient bien qu'il soit là en même temps que les acheteurs, et des éleveurs dont le calendrier de production dépend de la pousse de l'herbe. Négociation entre des estivants qui veulent s'incorporer du naturel, mais jugent à travers un référent basé sur l'industriel, et des bouchers qui, en montant au créneau pour défendre l'excellence du produit, se qualifient eux-mêmes dans une démarche de qualité (Erumel, 2003).

Nous avons tenté de faire l'analyse de la complexité et des contraintes. Elles sont tout d'abord environnementales : utiliser les parcours plutôt que les prairies et faire pousser l'agneau en même temps que l'herbe. C'est à ces conditions-là que les éleveurs ont le soutien du Parc, et qu'ils s'engagent dans une démarche en conformité avec leur désir de ne pas «artificialiser» leur élevage.
Les contraintes sont aussi celles du marché : mieux valoriser pour produire moins et mieux. Seuls les bouchers peuvent éduquer à la différence des consommateurs aux attentes très normées. En se faisant les médiateurs entre le client, le Parc et les producteurs, ils jouent un rôle-clé. Les éleveurs, pour maintenir leur autonomie par rapport à des filières plus classiques qui leur imposeraient une uniformité et une artificialisation de leur mode de production, acceptent de bas revenus et sont contraints à une renégociation continuelle avec de nouveaux intermédiaires. Ils sont «bio » sans nécessairement s'en prévaloir, mais affirment avec fierté ne pas faire du «bio de garage ", comme d'autres qui se contentent de respecter à la lettre les obligations du label en élevant des animaux qui ne sortent pas, avec des aliments bio produits ailleurs. Tout en vendant leur agneau au sein du Parc dans une filière, les éleveurs font de la vente directe et s'inscrivent dans la dynamique d'une association distribuant des produits de terroir. Pourtant, l'inadéquation entre le calendrier des touristes et celui de la production reste un problème majeur. Les démarches individuelles des éleveurs pourraient compromettre la survie du collectif, tandis que la production d'agneaux plus précoces mettrait en danger l'ensemble du système de production. Peut-on ajouter, à la tension entre produire et protéger, celle d'une distribution locale du produit?

\section{Références}

Aupetitgendre, M., Beisson, C., 1997. Rapport d'étude socioéconomique : condition de viabilité de la micro-filière Agneaux de Parcours, ISARA.

Bérard, L., Marchenay, P., 1995. Lieux, temps et preuves. La construction sociale des produits de terroir, Terrain, 24, 153-164.

Callon, M. (Ed.), 1989. La Science et ses réseaux, Paris, La Découverte.

Callon, M., Meadel, C., Rabeharisoa, V., 2000. L'économie des qualités, Politix, 52, 13, 521-556.

Chabert, J.-P., Lécrivain, É., Meuret, M., 1998. Éleveurs et chercheurs face aux broussailles, Le Courrier de l'environnement de l'INRA, 35, 5-12.

Conseil national de l'alimentation, 2002. Avis sur la Notion de qualité, élaboré sur le rapport du groupe de travail constitué par le CNA, présidé par Jean-Claude Olivier du collège des producteurs agricoles, Bertil Sylvander de l'INRA-UREQUA en étant le rapporteur, Avis $\mathrm{n}^{\circ} 36$, adopté le 26 mars 2002 par procédure écrite. Consultable et téléchargeable à l'url : http://www.agriculture.gouv.fr/spip/IMG/pdf/avis_36.pdf

Erumel, A., 2003. L'art des bergers et les moutons qualifiés : les figures sociales en jeu sur le marché agricole, Sociologie $d u$ travail, 45, 2, 169-189.

Friedberg, C., Cohen, M., Mathieu, N., 2000. Faut-il qu'un paysage soit ouvert ou fermé? L'exemple de la pelouse sèche du causse Méjan, Natures Sciences Sociétés, 8, 4, 26-42. 
Lardon, S., Osty, P.-L., Triboulet, P., 1995. Éleveurs et élevages du causse Méjan. Dynamique de la mise en valeur et contrôle de l'espace, in Bonniol, J.-L., Saussol, A. (Eds), Grands Causses, nouveaux enjeux, nouveaux regards : hommage à Paul Marres. [Actes du colloque de Millau-Meyrues, 21-23 octobre 1993], [Millau], Fédération pour la vie et la sauvegarde du pays des Grands Causses, 219-242.

Lepart, J., Marty, P., Rousset, O., 2000. Les conceptions normatives du paysage. Le cas des Grands Causses, Natures Sciences Sociétés, 8, 4, 16-25.

Long, A., Van der Ploeg, J.D., 1994. Styles of farming: An introductory note on concepts and methodology, in Van der Ploeg, J.D., Long, A. (Eds), Born from within: Practice and perspective of endogenous rural development, Assen, Van Gorcum, 7-30.

PNC, 2000. Programme d'aménagement 2000-2006, Parc national des Cévennes.

PNC, 2003. Rapport d'activité 2003, Parc national des Cévennes. Consultable et téléchargeable à l'url : http://www.bsi.fr/ pnc/Le_Parc/Rapport_activite_PNC_2003.pdf

Reçu le 2 mars 2005. Accepté le 8 septembre 2005.
Prache, S., Thierez, M., 1990. Production d'agneaux à l'herbe. Élevage ovin, le savoir-faire et l'enseignement agricole, Paris, Ministère de l'Agriculture et de la Forêt.

Rousset, O., Lepart, J., 1999. Évaluer l'impact du pâturage sur le maintien des milieux ouverts. Le cas des pelouses sèches, Fourrages, 159, 223-235.

Sainte-Marie, C. de, Prost, J.-A., Casabianca, F., Casalta, E., 1995. Construction sociale de la qualité, in Nicolas, F., Valceschini, E. (Eds), Agro-alimentaire : une économie de la qualité, Paris, INRA/Economica, 185-208.

Stassart, P., 2003. Produits fermiers : entre qualification et identité, Bruxelles, PIE-Peter Lang.

Sunt, C., 2002. Réinventer les circuits courts : une nécessité pour la bio, Fruits Oubliés, 30, 4/2002 ; http://perso.wanadoo.fr/ association.fruits.oublies/textfond/Sauvbio01.html

Thévenot, L., 1995. Des marchés aux normes, in Allaire, G., Boyer, R. (Eds), La Grande Transformation de l'agriculture : lectures conventionnalistes et régulationnistes, Paris, INRA/Economica, 33-51. 